\title{
V2282 Sgr Revisited
}

\author{
Roberto Nesci ${ }^{1}$, Corinne Rossi ${ }^{2}$, Antonio Frasca ${ }^{3}$, Ettore Marilli ${ }^{3}$, Paolo Persi ${ }^{1}$, Nicola Cornero ${ }^{4}$ \\ ${ }^{1}$ INAF-IASF, via Fosso del Cavaliere 100, 00133 Roma, Italy \\ ${ }^{2}$ University La Sapienza, P.le A. Moro 2, 00186 Roma, Italy \\ ${ }^{3}$ INAF/OACt, Catania, Italy \\ ${ }^{4} \mathrm{CDSO}$, Aosta, Italy
}

Corresponding author: roberto.nesci@iaps.inaf.it

\begin{abstract}
The nature of V2282 Sgr is examined on the basis of several multiband observations: a 20 years long I-band light curve of V2282 Sgr obtained from archive photographic plates of the Asiago and Catania Observatories; a CCD R-band light curve obtained at Cornero Observatory; JHK photometry from 2MASS and UKDISS; Spitzer IRAC and MIPS images; optical spectra from Loiano Observatory; X-ray flux from CHANDRA. The star has a K-type spectrum with strong emission lines and is irregularly variable at all wavebands. The overall evidences suggest that V2282 Sgr is a Pre Main Sequence star with an accretion disk.
\end{abstract}

Keywords: variable stars - pre-main-sequence - stellar evolution.

\section{Introduction}

The M20 (Trifid) nebula is a spectacular star forming region of the Milky Way: it is projected in the sky over the open star cluster NGC 6514, and its distance has been estimated by several authors, ranging from $1.4 \mathrm{kpc}$ [6] up to $2.7 \mathrm{kpc}$ [1]. The variable V2282 Sgr was mainly studied by [5] who reported an amplitude of about 0.5 mag in the $I$ band and classified it as a possible Orion type variable. The star is positionally consistent with the soft X-ray source CXOM20 180216.8-230347 detected by CHANDRA in M20 [9]; it appeared not to be variable in X-rays, with a flux level of $0.167 \mathrm{c} / \mathrm{s}$, and therefore was not further discussed in that paper. It is also consistent with a SPITZER infrared source of the mid-infrared survey of the M20 by [10], classified as a proto-stellar source (Class I/0 n.16) without comments about a possible optical counterpart. A nebular variable (either of the T Tau, RW Aur or T Ori type) is believed to be a Pre Main Sequence (PMS) star already partially clear of its originating circumstellar envelope [2]: this seems at odds with classification as a Class I/0 object, which is a still deeply embedded source. For this reason we decided to explore in more detail its nature.

\section{Photographic Light Curve}

We measured the magnitude of V2282 Sgr on 25 plates with I-N emulsion and RG650 filter taken with the 65/90/235 Schmidt telescope of the Asiago Observatory, and on 109 (I-N + RG650) plates of the 40/40/120 Schmidt of the Catania Observatory. Plates were digitized with an Epson 1680 Pro scanner in transparency mode at 1600 dpi. A sequence of comparison stars was defined selected from the GSC2.3.2 catalog, using the $\mathrm{N}$ band magnitudes (see Fig. 1 and Table 1). Aperture photometry was made with IRAF/apphot. The photometric accuracy of our magnitudes ranges from $0.08 \mathrm{mag}$ at $I=13$ to $0.18 \mathrm{mag}$ at $I=14.5$ : fainter stars have larger dispersion, as expected. The rms deviation of the values of $N$ magnitude for V2282 Sgr was $0.25 \mathrm{mag}$ in the Asiago and $0.34 \mathrm{mag}$ in the Catania datasets, substantially larger than the faintest comparison star. The resulting overall light curve of the Asiago and Catania observations is shown in Fig. 2. V2282 Sgr shows substantial variations up to 1 magnitude, with an average level $N=14.0$. 


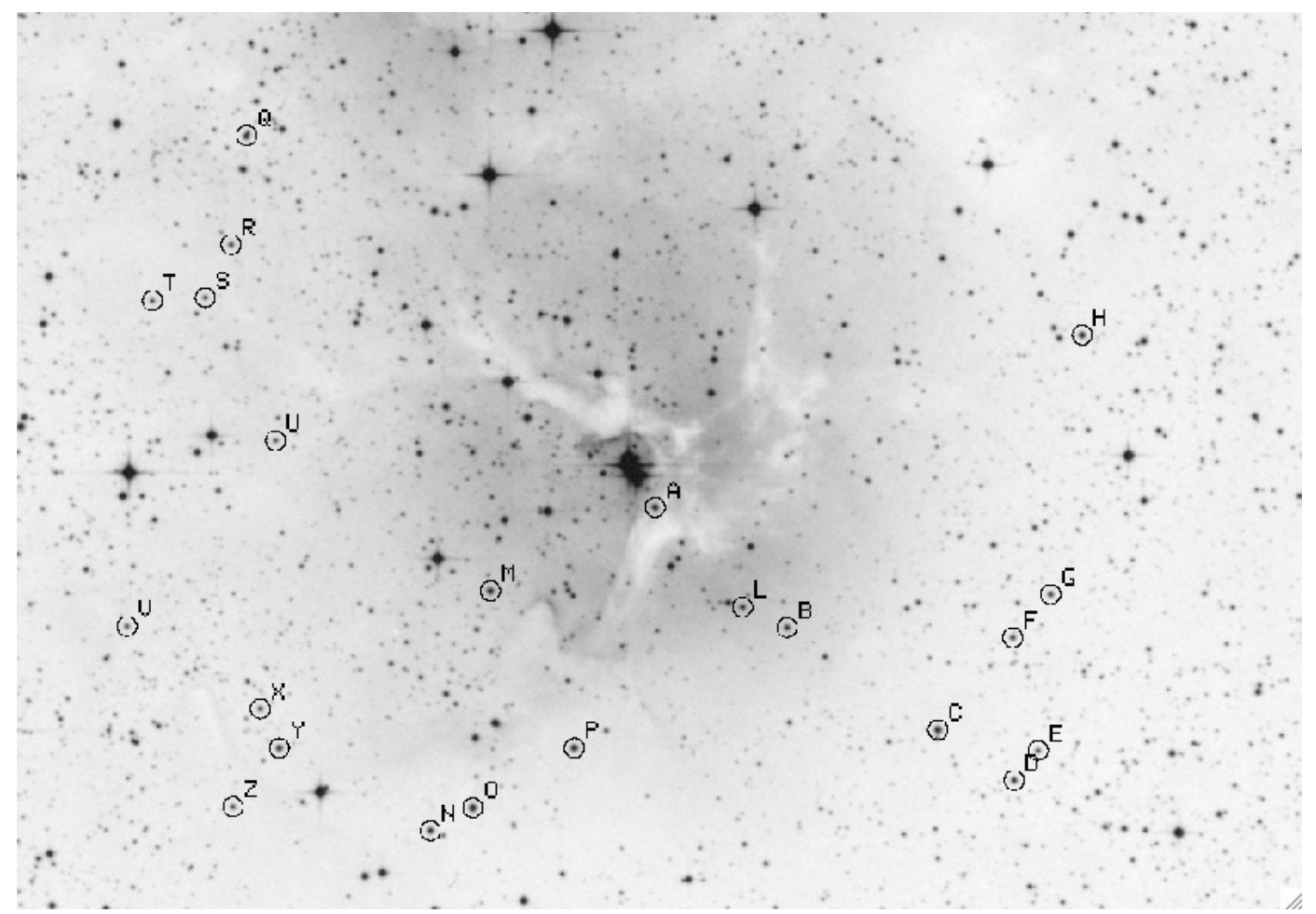

Figure 1: Finding chart of V2282 Sgr from a POSS II plate with IV-N emulsion. The variable is marked with L. Comparison stars are labelled. The field is 12 arcmin wide in DEC, North is up and East to the left.

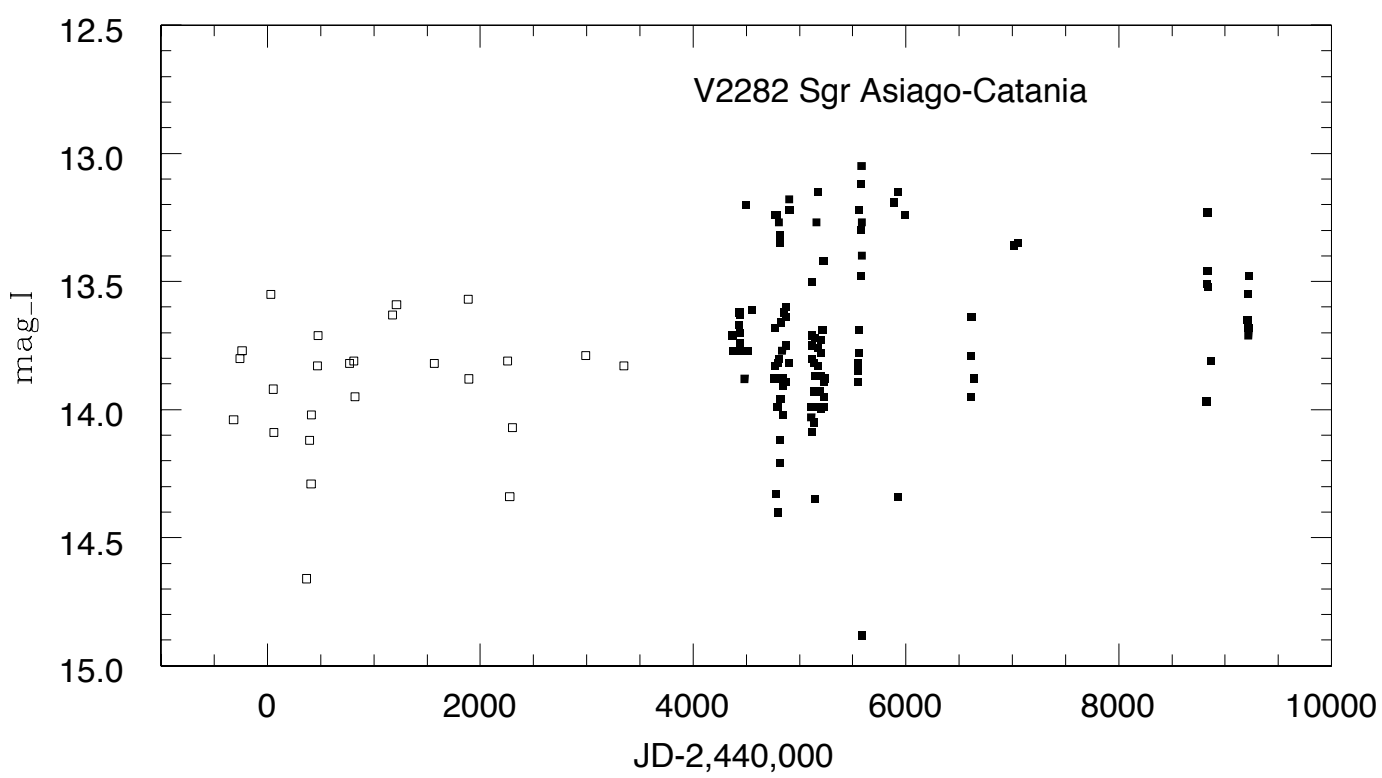

Figure 2: Light curve in the $I$ photographic band of V2282 Sgr from the Asiago (open squares) and Catania (filled squares) archives. Due to its southern sky position the coverage shows a marked seasonal bias. 


\section{CCD Observations}

CCD images of M20 were taken at the Cornero Observatory $^{1}$ with a $30 \mathrm{~cm} \mathrm{F/6.5} \mathrm{Schmidt-Cassegrain} \mathrm{telescope}$ and an $R_{\mathrm{C}}$ filter in 19 different nights, from June to October 2009. The star showed an oscillating behaviour, with an amplitude of about one magnitude (see Fig. 3.) The time interval between a minimum and a maximum may be as short as 10 days, so that the loose time sampling of historic plates may underestimate the actual variability range.

Furthermore, we obtained with the BFOSC instrument at the Loiano $1.52 \mathrm{~m}$ telescope a $B, V$ and $R$ photometric point on 2011 and an $R$ one in 2012. The star was at $B=17.60, V=16.31, R=14.70$ on 2011 and $R=14.40$ on 2012 ; both $R$ values are within the variability range of the light curve of 2009.

\section{Optical Spectroscopy}

With the BFOSC instrument we also secured two spectra of the star (on 2011-08-02 and 2012-07-18) at 4 $\AA /$ pixel dispersion (resolution $\sim 12 \AA$ ). The spectra were reduced using the IRAF reduction package, following the standard procedures. Due to the large airmass no reliable flux calibration could be obtained. The one dimensional spectrum of V2282 Sgr shows a number of emission lines which are most probably formed in a region physically close to the star. The two spectra taken at a distance of one year from each other look rather similar. The only remarkable difference is that the $[\mathrm{N}$ II] $6584 \AA$ line is not apparent in July 2012 .

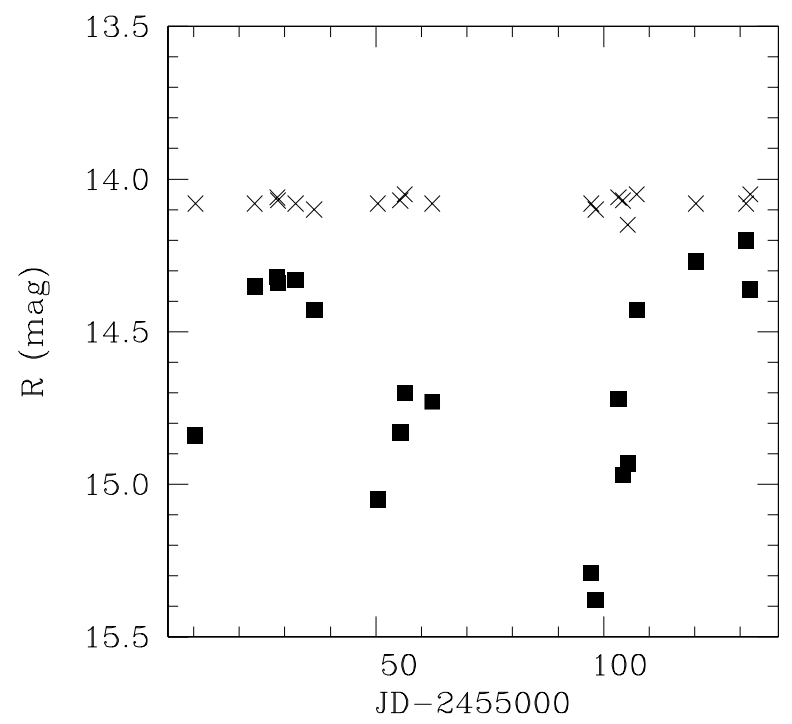

Figure 3: The light curve of V22822 Sgr from CCD images of the Cornero Observatory. Crosses are a comparison star, filled squares are the variable.
Table 1: Adopted comparison stars sequence

\begin{tabular}{ccccccc}
\hline RA & DEC & Id & $N$ & $B$ & $V$ & $F$ \\
\hline 270.591 & -23.040 & A & 12.69 & 13.99 & 13.37 & - \\
270.560 & -23.068 & B & 13.52 & 15.47 & 14.73 & - \\
270.522 & -23.091 & C & 12.26 & 13.15 & 12.96 & 12.66 \\
270.503 & -23.103 & D & 13.02 & - & - & 13.72 \\
270.497 & -23.096 & E & 13.49 & - & - & 13.82 \\
270.503 & -23.070 & F & 13.29 & 14.81 & 14.44 & 13.57 \\
270.494 & -23.061 & G & 13.27 & - & - & 13.93 \\
270.486 & -23.001 & H & 12.63 & 13.15 & 12.96 & 12.82 \\
270.633 & -23.059 & M & 12.49 & 14.40 & 13.73 & - \\
270.647 & -23.114 & N & 12.82 & - & - & 13.37 \\
270.637 & -23.108 & O & 12.22 & 12.44 & 12.29 & 12.36 \\
270.612 & -23.095 & P & 12.52 & 13.71 & 13.15 & 12.87 \\
270.692 & -22.955 & Q & 13.11 & - & - & - \\
270.696 & -22.980 & R & 13.68 & 15.36 & 14.55 & - \\
270.703 & -22.992 & S & 14.13 & - & - & - \\
270.716 & -22.993 & T & 14.21 & - & - & 14.38 \\
270.685 & -23.025 & U & 14.40 & 14.52 & - & - \\
270.722 & -23.067 & V & 13.89 & 15.35 & 14.53 & - \\
270.689 & -23.086 & X & 13.80 & - & - & - \\
270.685 & -23.095 & Y & 12.85 & 14.42 & 13.72 & 13.24 \\
270.696 & -23.108 & Z & 13.82 & 15.40 & 14.71 & - \\
\hline & & & & & &
\end{tabular}

Table 2: Emission lines in V2282 Sgr

\begin{tabular}{ccc}
\hline wavelength & ion & $E W(\AA)$ \\
\hline 6584 & {$[\mathrm{~N} \mathrm{II}]$} & -5 \\
6563 & $\mathrm{H} \alpha$ & -20 \\
6548 & {$[\mathrm{~N} \mathrm{II}]$} & $-0.6::$ \\
5007 & {$[\mathrm{O} \mathrm{III}]$} & -10 \\
4959 & {$[\mathrm{O} \mathrm{III}]$} & -5 \\
4861 & $\mathrm{H} \beta$ & -16 \\
4363 & {$[\mathrm{O} \mathrm{II}]$} & $-6::$ \\
4340 & $\mathrm{H} \gamma$ & -15 \\
\hline
\end{tabular}

The emission lines in the stellar spectrum are listed in Table 2, together with their equivalent width $(E W)$. We remark that doing the sky subtraction from the raw spectrum of the star all the night-sky emission lines disappeared, while the [O III] and Balmer emission lines remained, so we are strongly convinced that they are real.

\footnotetext{
${ }^{1}$ http://www.cdso.it
} 
Among the absorption lines, the most prominent are the NaI D doublet $(6.5 \AA)$, the MgI b triplet $(3.3 \AA)$, the blends of several atomic lines centered at 5269 and $6497 \AA$, the CaI $4227 \AA$ and the G band, indicating an intermediate G-K spectral type. The NaI D doublet is very strong, compared to the typical value $(2.5 \AA)$ of the main sequence or giants G-K stars, suggesting the presence of substantial interstellar/circumstellar matter, as expected in a PMS star.

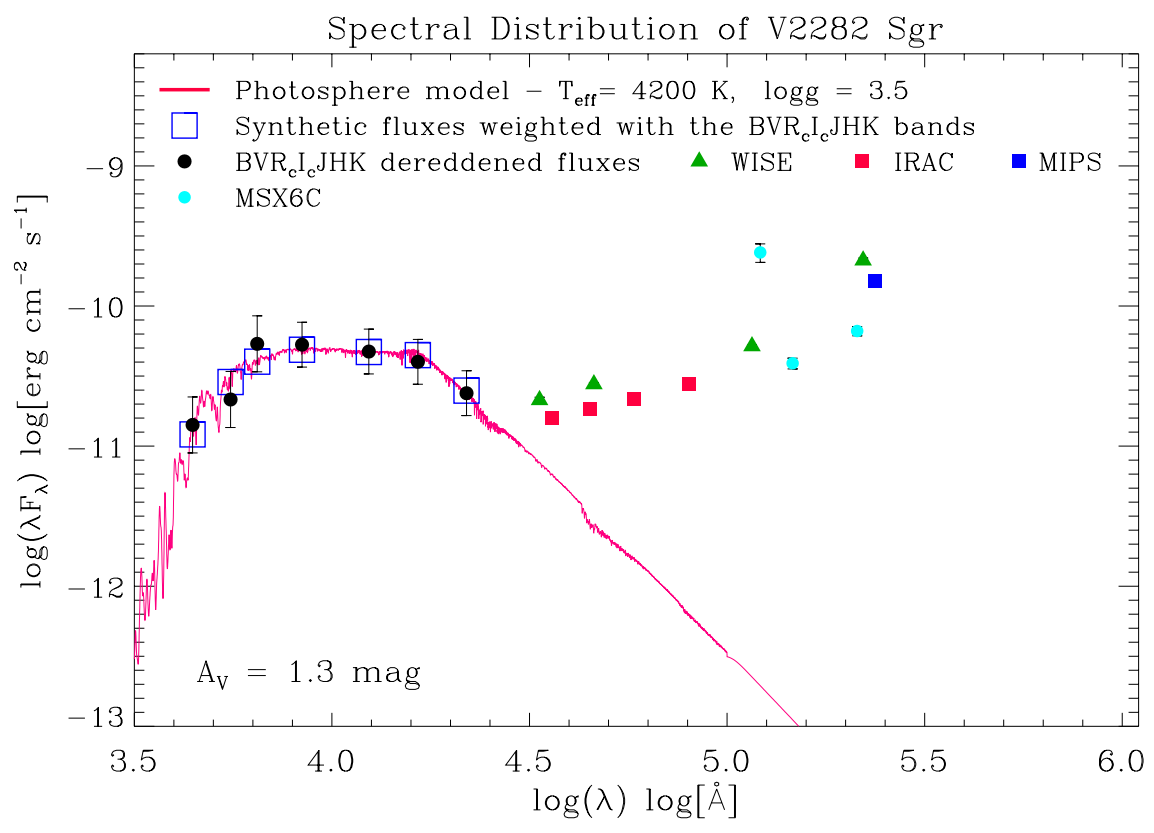

Figure 4: SED of V2282 Sgr from photometric de-reddened $\left(A_{V}=1.3 \mathrm{mag}\right)$ data. The best-fitting NextGen synthetic spectrum with $\mathrm{T}=4200 \mathrm{~K}$ and $\log g=3.5$, is over-plotted with a continuous line.

To gain information about the stellar photosphere, we fitted the observed SED with low-resolution synthetic spectra calculated from the NextGen model atmospheres [3]. We fixed the star distance and extinction to the values $d=1.7 \mathrm{kpc}$ and $A_{V}=1.3 \mathrm{mag}$ reported by [4] and we let $T_{\text {eff }}$ and $\log g$ free to vary, adopting a standard extinction law $A_{V}=3.1 E(B-V)$. This interstellar absorption is consistent with the typical value of $A_{V}=0.8 \mathrm{mag} / \mathrm{kpc}$ and a distance of $1.7 \mathrm{kpc}$.

The fit was limited to the photometric bands from $B$ to $J$, because afterwards the photospheric flux could be significantly contaminated by an IR excess. The result of the SED fit is shown in Fig. 4 where the optical and near-IR fluxes have been corrected for the extinction and the error bars include the average variation of \pm 0.5 mag observed both in $R$ and $I$ bands. The best-fit model is that with $\log g=3.5$ and $T_{\text {eff }}=4200 \mathrm{~K}$. With these parameters, a radius of about $4.8 R_{\text {sun }}$ would be derived for the star at the adopted distance. The observed flux appears to be dominated by the stellar photosphere up to the $K$ band, while an IR excess, likely related to a circumstellar disk, is quite evident longward of $3 \mu \mathrm{m}$.
To quantify how much the derived temperature is sensitive to the adopted $A_{V}$ we performed several tests: two extreme cases are reported below. Adopting $A_{V}=0.5$ gives $T_{\text {eff }}=3800 \mathrm{~K}, \log g=2.5$ and a radius $5.1 R_{\text {sun }}$ : such a low temperature is not compatible with the observed spectrum, which shows no molecular bands. Adopting a substantially higher value $\left(A_{V}=3.0\right)$ gives $T_{\text {eff }}=5600 \mathrm{~K}, \log g=3.5$ and a radius $4.3 R_{\text {sun }}$ : this is still compatible with the observed spectral features but produces somewhat larger residuals than the $A_{V}=1.3$ case. The derived stellar temperature is clearly an increasing function of the assumed absorption.

We remark that the actual absorption is the sum of the interstellar absorption, the circumstellar absorption and the possible contribution from the M20 nebula whose outskirts include the star.

A measure of the actual absorption is not easy because the star spectrum is peculiar: in particular the $E W$ of the MgI triplet is low for a G5 star $(5600 \mathrm{~K})$, and even more so for a K0 $(4400 \mathrm{~K})$ one: it would be typical of a Main Sequence F5 star, which is too hot for the observed spectral features. This suggests that a strong 
veiling is present in the spectrum, as indicated also by the presence of strong Balmer emission lines. Therefore we cannot reliably derive the amount of absorption by comparing the intrinsic $\mathrm{B}-\mathrm{V}$ with the observed one.

The $E W$ of the NaI D doublet is $6.5 \AA$, so it is well in the saturated part of the relation between E.W. and $\mathrm{E}(\mathrm{B}-\mathrm{V})$ by $[8]$ and cannot give an accurate estimate of the color excess, save indicating that it must be substantially larger than the simple interstellar one.

\section{Infrared Observations}

To better understand the nature of V2282 Sgr we considered the $J, H$ and $K$ magnitudes taken at different epochs from 2MASS, DENIS, UKIDSS catalogues and from [10] (see Table 3). From this Table it appears evident the near IR variability of the star.

Table 3: Near Infrared Photometry

\begin{tabular}{lllll}
\hline \hline Date & $J$ & $H$ & $K$ & Note \\
\hline $1998 / 06 / 14$ & 12.74 & 12.02 & 11.54 & 2MASS \\
$1996 / 04 / 01$ & 12.90 & $\ldots$ & 11.51 & DENIS \\
$2004 / 08 / 23$ & 12.99 & 12.26 & 11.93 & 200inch \\
$2006 / 07 / 23$ & 15.71 & 14.21 & 12.78 & UKIDSS \\
\hline
\end{tabular}

Spitzer/IRAC images between 3.6 and $8 \mu \mathrm{m}$ were taken in three different epochs. We performed the photometry of the star using IRAF/apphot with the same aperture size of [10]. The star was significantly variable, mainly at shorter wavelengths, enforcing the evidence of its PMS nature.

A bolometric luminosity, with an uncertainty of about one magnitude, may be computed from the infrared Spitzer, JHK, and our optical data. We derived a value of $4.1 L_{\text {sun }}$ in the range from $B$ to $K$ and 24.3 $L_{\text {sun }}$ in the range from $B$ up to $24 \mu \mathrm{m}$, quite reasonable values for a PMS star.

\section{Conclusions}

V2282 Sgr can be classified as a probable Class I PreMain Sequence star associated with an accreting circumstellar disk. Its observed optical spectrum is consistent with a late $\mathrm{G}$ type star. Its long-term irregular optical variability, typical of accreting $\mathrm{T}$ Tauri stars, can be due to a combination of stellar rotation and circumstellar dust occultation, while the observed transient near-IR excess arises from a combination of variable extinction and changes in the inner accretion disk. Its X-ray luminosity $\left(7.6 \times 10^{32} \mathrm{erg} \mathrm{cm}^{-2} \mathrm{~s}^{-1}\right)$ is compatible with the value for PMS with mass $\sim 2 M_{\text {sun }}$ [7].

One may wonder if V2282 Sgr is actually a foreground object unrelated to the Trifid nebula: we think that the strong high-excitation [O III] and N[II] lines in its spectrum prove the presence of hot gas in front of the star, which cannot be heated by the low temperature of the stellar photosphere and is therefore related to the M20 nebula.

\section{Acknowledgement}

R. Nesci thanks the Direction of the Asiago Observatory for hospitality during the scanning of the archive plates.

\section{References}

[1] Cambresy, L., Rho, J., Marshall, D.J., Reach, W.T., 2011 A\&A 527, 141

[2] Glasby J.S., The nebular variables, Pergamon Press, 1974

[3] Hauschildt, P. H., Allard, F., Ferguson, J., Baron, E., \& Alexander, D. R. 1999, ApJ, 525, 871 doi:10.1086/307954

[4] Lynds, B. T., Canzian, B. J., \& O'Neil, E. J., Jr. 1985, ApJ 288, 164 doi:10.1086/162775

[5] Maffei, P., 1963, MemSAIt 34, 441

[8] Munari, U., Zwitter, T., 1997, A\&A 318, 269

[6] Ogura, K., \& Ishida, K. PASJ 1975, 27, 119

[7] Preibisch T. 2007, MemSAIt 78, 1

[9] Rho, J., Ramirez, S.V., Corcoran, M.F., Hamaguchi, K., Lefloch, B., 2004, ApJ 607, 904 doi:10.1086/383081

[10] Rho, J., Reach, W.T, Lefloch, B., Fazio, G.G, 2006, ApJ 643, 965 doi:10.1086/503245 